\title{
Reflets
}

Revue d'intervention sociale et communautaire

\section{Hommage à Richard Carrière, directeur fondateur de Reflets}

\section{Madeleine Dubois}

Volume 18, numéro 1, printemps 2012

URI : https://id.erudit.org/iderudit/1012328ar

DOI : https://doi.org/10.7202/1012328ar

Aller au sommaire du numéro

\section{Éditeur(s)}

Reflets, Revue d'intervention sociale et communautaire

ISSN

1203-4576 (imprimé)

1712-8498 (numérique)

Découvrir la revue

Citer ce document

Dubois, M. (2012). Hommage à Richard Carrière, directeur fondateur de Reflets. Reflets, 18(1), 9-11. https://doi.org/10.7202/1012328ar d'utilisation que vous pouvez consulter en ligne.

https://apropos.erudit.org/fr/usagers/politique-dutilisation/ 


\section{Hommage à Richard Carrière, directeur fondateur de Reflets}

Madeleine Dubois, membre du comité de direction de Reflets

Publié à l'automne 1995, le premier numéro de Reflets a vu le jour grâce à la vision et à la détermination d'un groupe de professeurs de l'Université Laurentienne et de l'Université d'Ottawa et de quelques-uns de leurs collègues œuvrant dans des organismes communautaires en Ontario français. Richard Carrière, professeur à l'École de service social de l'Université Laurentienne, fut l'un des instigateurs principaux de cette initiative. Cette même passion qui l'avait habité lors de la fondation de Reflets, Richard a continué de la nourrir et de la partager au cours des quinze ans pendant lesquels il a dirigé la revue. Avec sa retraite de l'enseignement, Richard quitte aussi la direction de Reflets. Nous voulons le remercier et souligner sa remarquable contribution à la création, à l'essor et au succès de la revue qui a permis, au cours des années, de donner un forum à de nombreux chercheurs, chercheuses et à des intervenants des milieux communautaires, de mettre en lumière de nombreux enjeux concernant les divers contextes minoritaires et d'en débattre.

C'est à l'invitation de Richard qu'en 1992, une vingtaine de personnes se réunissent à l'Université Laurentienne afin de discuter des tenants et aboutissants inhérents à la création d'une revue francophone portant sur l'intervention sociale en Ontario. Les défis entourant la mise sur pied d'une revue sont nombreux, mais l'équipe qui compose le comité de direction est mue par le désir de se doter d'un mécanisme pour alimenter la réflexion, la recherche et la diffusion d'information portant sur l'intervention sociale et communautaire en Ontario français. Une des particularités - et également des défis — réside dans 
le désir d'ouvrir les possibilités de publication non seulement aux professeurs et représentants des milieux universitaires, mais également aux intervenantes et intervenants communautaires qui innovent et adaptent leurs pratiques aux particularités régionales et à la diversité de l'Ontario français. Pour atteindre cet objectif, des représentants des milieux communautaires se joignent à ceux du milieu universitaire pour former le comité de direction. On crée des rubriques pour encourager la pluralité des écrits : l'Entrevue qui vise à donner la parole à une ou des personnes dont l'engagement ou la recherche sont liés au thème de chaque numéro; le Dossier, qui comprend des articles de fond soumis à une évaluation par les pairs et répondant aux exigences de publication universitaires; Des pratiques à notre image, qui donne la possibilité aux intervenants de présenter et d'analyser des expériences de pratique adaptées à leurs milieux respectifs; $A u x$ études, qui présente les résumés de mémoires ou de thèses d'étudiants diplômés des programmes francophones de service social de $2^{\mathrm{e}}$ ou $3^{\mathrm{e}}$ cycle des universités ontariennes; et finalement Lu pour vous, qui propose des comptes-rendus de livres ou de rapports pertinents aux pratiques d'intervention en contextes minoritaires francophones.

Atteindre les objectifs établis pour Reflets exige de cultiver un réseau tant dans les milieux universitaires que communautaires. Plusieurs interlocuteurs de ces milieux seraient en mesure d'illustrer à quel point Richard a toujours su profiter de toutes les occasions pour faire la promotion de la revue. Que ce soit lors de sa participation à un colloque universitaire ou à l'Assemblée générale annuelle d'une association provinciale ou régionale, Richard endossait toujours son chapeau d'ambassadeur de Reflets et il savait tirer profit de toutes les occasions pour prendre la parole de façon spontanée et persuasive dans le but de faire connaître la revue, invitant les participants et les participantes à y contribuer des articles ou à s'y abonner.

Créer et maintenir une revue telle que Reflets exige aussi évidemment du soutien financier et là encore, Richard ne ratait aucune opportunité pour faire appel aux bailleurs de fonds potentiels. Son approche éloquente et convaincante face aux politiciens, aux fonctionnaires ou aux administrateurs 
universitaires a engendré du soutien financier inestimable pour l'essor de la revue, que ce soit grâce à des " fonds de tiroir » ou à de modestes montants récurrents. Nous n'avons aucun doute que Richard nous enjoindrait, dans le cadre de cet hommage lui étant adressé, de remercier très chaleureusement tous les bailleurs de fonds qui ont soutenu Reflets au cours des années et qui continuent de le faire.

La vision et la détermination de Richard, le sens d'humour avec lequel il recentrait les débats les plus houleux au sein de l'équipe et son optimisme contagieux ont laissé une marque indélébile auprès de ses collègues du comité de publication qui perpétuent l'œuvre et la vision de Reflets. Nous te saluons et te remercions, Richard, de ton apport remarquable à la création d'un médium de communication bien vivant pour les communautés francophones en contextes minoritaires et nous te souhaitons une retraite longue et des plus agréable! 